\title{
Geometrische Aufgaben
}

$\mathrm{nach}$

\section{der Methode der Griechen}

\author{
bearbeitet
}

vo $n$

Dr. W. A. $D$ i e $s t$ e $r$ w $g$ ord. Professor der Mathematik auf der königl. preufes Rheinuniversität:

Mit XIV. Kupfertafeln.

$$
\begin{aligned}
& \text { B e } 1 \text { i n } \\
& \text { bey Georg Reimex } \\
& 1825 \text {. }
\end{aligned}
$$





\title{
Den Herren Professoren
}

\author{
M. BLAND \\ i n $C$ a m b ridge,
}

IO. WILH. CAMERER i n $S$ t $u t g$ a $t$,

KARL FR. HAUBER i n $M$ a $\mathbf{l}$ b r o n $n$,

\section{IOHN LESLIE}

i n $\mathbf{E} d$ i n b u $\mathbf{r}$,

den g.ründlichen Kennern

der Geometrie der Griechen,

mit ausgezeichneter Hochachtung

$$
\text { gew id mot }
$$

von dem Verfasser. 
